\title{
Lawn Tennis Studied From The Medical Point of View
}

\author{
(Report No. 3) \\ By \\ R. Sakurai. \\ From the Orthopaedic Clinic of the Nihon Medical College, Tokyo, Japan. \\ (Director: Prof. Dr. K. Saito.)
}

\section{Introduction.}

In 1935, a study of the arms and chests of fifty-four leading temis players in Japan was begun. The two preceding reports dealt with the study of the upper limbs and the energy expenditure involed in playing lawn temis, respectively. This report concerns an aualysis of the X-ray findings of the chest.

\section{Procedure.}

The fifty-four subjects used in this study were among the leading lawn temis players in Japan in 1935. The age range was from eighteen to forty-nine years. The number of years of continuous participation was two to thirty years with an average of twelve years. The age at which temis was begun ranged from eight to twenty years with an average of fifteen years. Thus, I believe that the range of ages and the period of activity warrants the assumption that this is a fairly representative study.

A ventro-dorsal $\mathrm{X}$-ray photograph was made using a focal distance of two meters and the lower surface of the spinous process of the fifth dorsal vertebra as the point of focus while the subjects stood with shoulders raised forwards. Each picture was taken when the subject held his breath at the end of a full inspiration after a series of deep breaths. In this stridy the aualysis follows the procedure of Prof. Dr. Saito and Dr. Aikawa whose research took up a study of healthy individuals engaged in all walks of life whether athletes or nonathletes.

\section{Observations.}

From an aualysis of the X-ray photographs the following may be said:

\section{Clavicle.}

A. "Begleitschatten" of the Clavicle. 
This is the "shadow" of the skin of the region between the clavicle and the sub-clavian groove. Among the players it was perceived in 46 cases $(86.8 \%)$; on both sides in 41 cases; the right only in 3 cases, and on the left in 2 cases. The above data coincides, in general, with the previous findings of Prof. Dr. Saito and Dr. Aikawa.

B. "Usurschatten" of the Clavicle.

Prof. Dr. Saito and Dr. Aikawa gave this name to a condition in which the lower surface of the clavicle has been roughened. This is brought about by the recurrence of any movement which causes friction between the head of the first rib and the clavicle. Such a condition was found in the right of 9 and on the left of 4 subjects. In the right of 7 cases and left of 4 there may be some doubt as to its authenticity. In general, there were more on the right than the left side.

Let us analyze the serve. As the racquent is swung downward backward and upward, the service arm shoulder is drawn forcefully backward and the other arm i raised aloft as the ball is tossed upward. Then, the elbow is bent and the racquet dropped behind the head. There $\mathrm{i}$; a trancfer of weingt from the bank font to the forward font as the arm and racquet are extended forward upward to contact the ball over the forward foot and brought diagonally downward left with a bending of the trunk on the follow through. Of course, there are many variations to the overhead service and the first and second services usually differ in speed but the movements involved are fundamentally the same. Thus (we can realize) that considerable friction takes place in each serve.

In the case of the overhead smash, a similar extonsion of the arm and downward swinging on the follow-through are used. There are many other strokes in which approximately the same movements take place, notably the forehand stroke.

The "Usurschatten" is more maked on the side of the service arm. Temnis is a game which necessitates a great amount of this type of activity, so in considering the occurence from the viewpoint of mechanics, it is to be expected.

C. Epiphyses at the Sternal End of the Clavicle.

In 26 of the 30 cases apparent both sides were clearly seen and 4 of one side only, 2 of which were on the right. It is interesting to note that between the ages of 19 and 26 years the condition occurred in o:er half the number while it did not exist at all in the case of 4 persons of 25 years of age and one person of 18 years. 
Table 1.

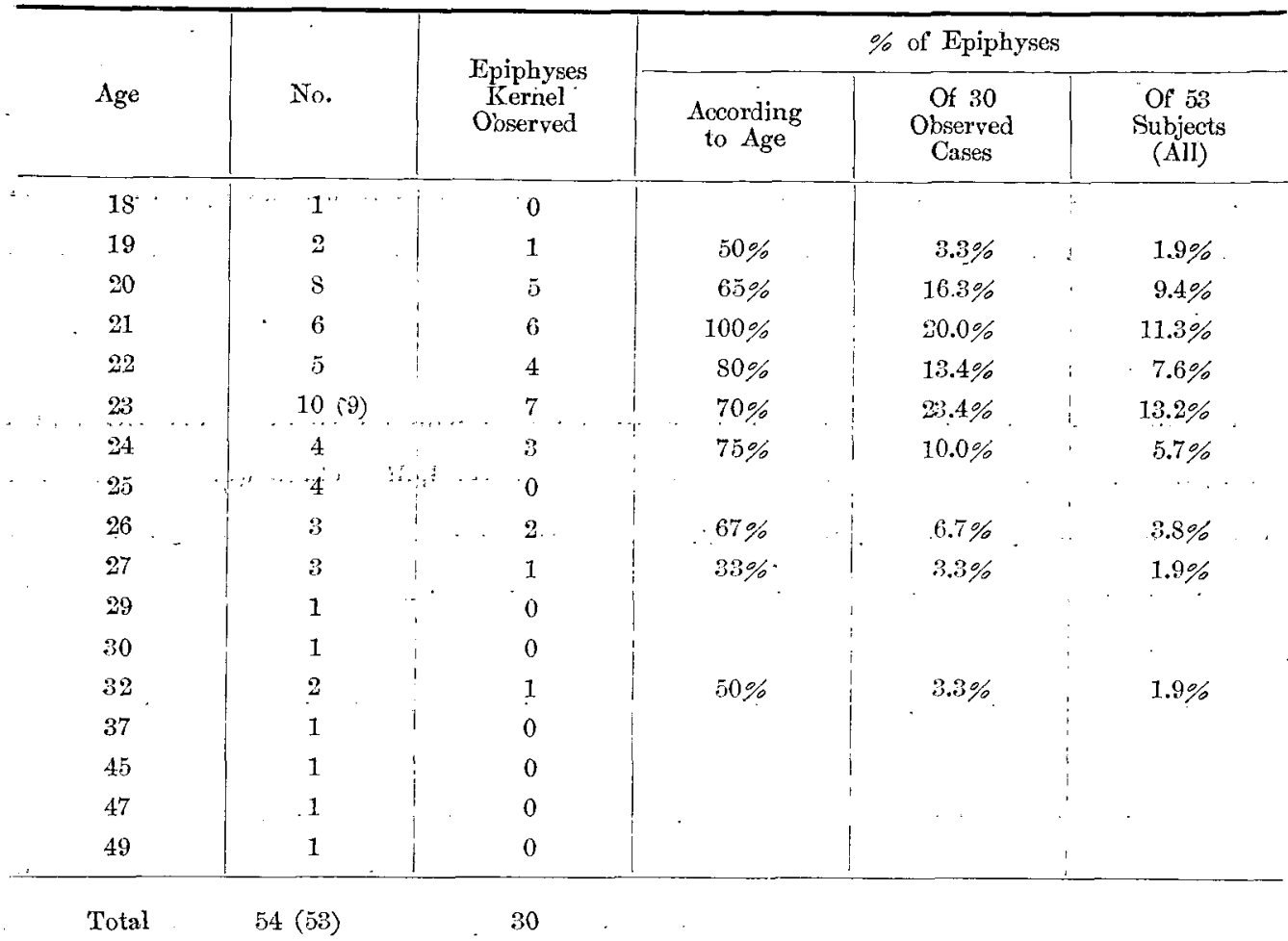

2. Rib́s.

A. Ossification of the Rib Cartilage.

It is generally accepted that the state of ossification of the rib cartilage is more complete with the advance of age. In the case of my own investigations $I$ found this true, but in the research of Prof. Dr. Saito and Dr. Aikawa some interesting exceptions to this "rule" were brought to light. That there is a greater degree of ossification in the case of the first cartilaginous process than in any of the others is also a well-kuown fact. No special relationship between the state of ossification and pathological changes of the lungs was fonnd. In 17 cases $(32.1 \%)$ a complete nssification of one or more ribs was apparent.

B. Rib Deformities and "Alignment".

In briefly reviewing the variations found a few interesting cases were as follows: " Case No. 1.

There was a widening of the left second rib from the angle toward the shaft and at the anterior side of the chest a marked uarrowing as though shaved =crescent shaped- then a return to normal width at the costal cartilage. 
The upper surface of the right second rib was rotgh and two centimeters beforeaching the costal cartilage there was a decided upward curve but the lower surface of the rib was normal. There were no indications of disease or fracture in either case.

Case No. 2.

About 3 centimeters from the angle toward the sternum there was an upward curve of both surfaces of the right second rib, then a return to normal width. No indications of disease or fracture were apparent.

Case No. 3.

The subject's thorax was small as a whole similar to a feminine thorax. The ribs had a decided downward angle, especially prominent in the lower half. There were no particular variations in the spine or diaphragma apparent in the $\mathrm{X}$-ray picture. Although there wereno indications disease, there was a lobular deformity of the right lung.

Case No. 4.

There was a "spine-shaped" process near the costal cartilage of the right second rib. Case No. 5.

The first and second ribs were joined together 3 centimeters from their heads for a short

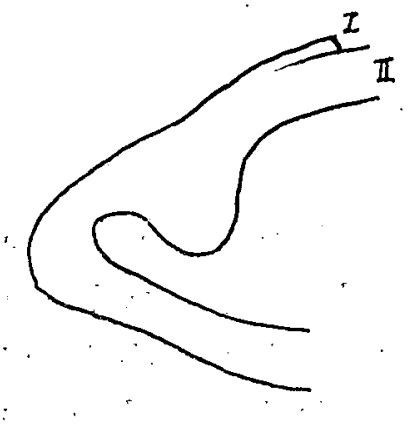
distance. The second 'rib continued on normally while the first rib widened at the point of separation and terminated with a downward angle.

Case No. 6.

"The upper half of the ribs "left" the spine horizontally and in extreme cases with an upward angle. From the " angle" the ribs continued toward the sternum with a decided dowuward angle. The above was a very exceptional case.

.It was interesting to note that the subject was one of the very best players in Japan.

\section{3. "Begleitschatten" of the Second Rib.}

In 27 cases. $(50.9 \%)$ the shadow of the pleura of the apex of the lungs was clearly seen; of which 12 (44.4\%). were on the right; 9 (33.3\%), the.left ; 6 (22.2\%) both sides., I shall divide them into 4 classes as indicated in the following diagram.

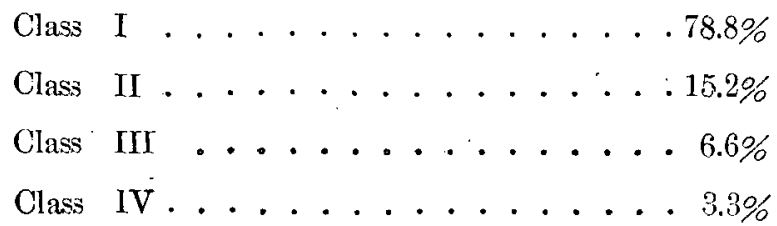




\section{Hair-line (Right middle lobe of the lung)}

They were apparent 33 in cases $(60.4 \%)$. "With the exception of specialc ases, the hair-lines did not indicate any diseased condition. The research of Prof. Dr. Saito and Dr. Aikawa confirmed Dr. Schin i's theory that these are normal. 'The findings of this study further verified this theory.

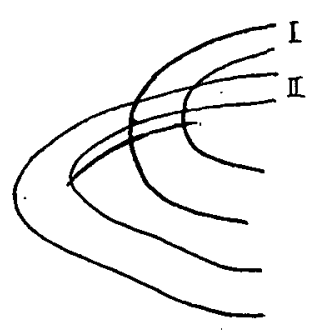

〔I\}

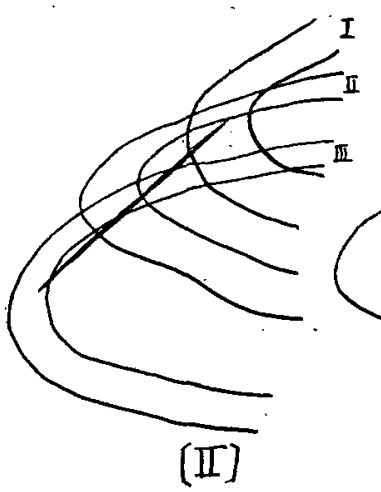

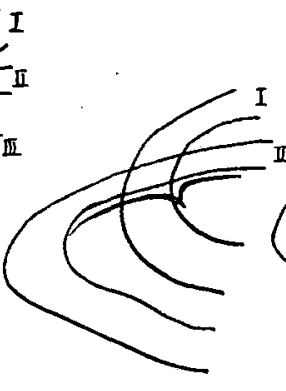

[iil]

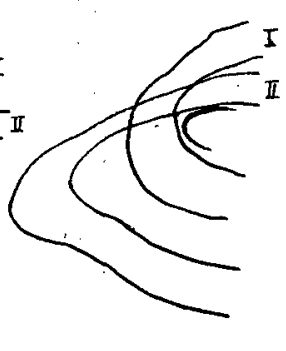

$(\mathbb{V})$

In this study the hair-lines have been divided into classes according to the following diagram :

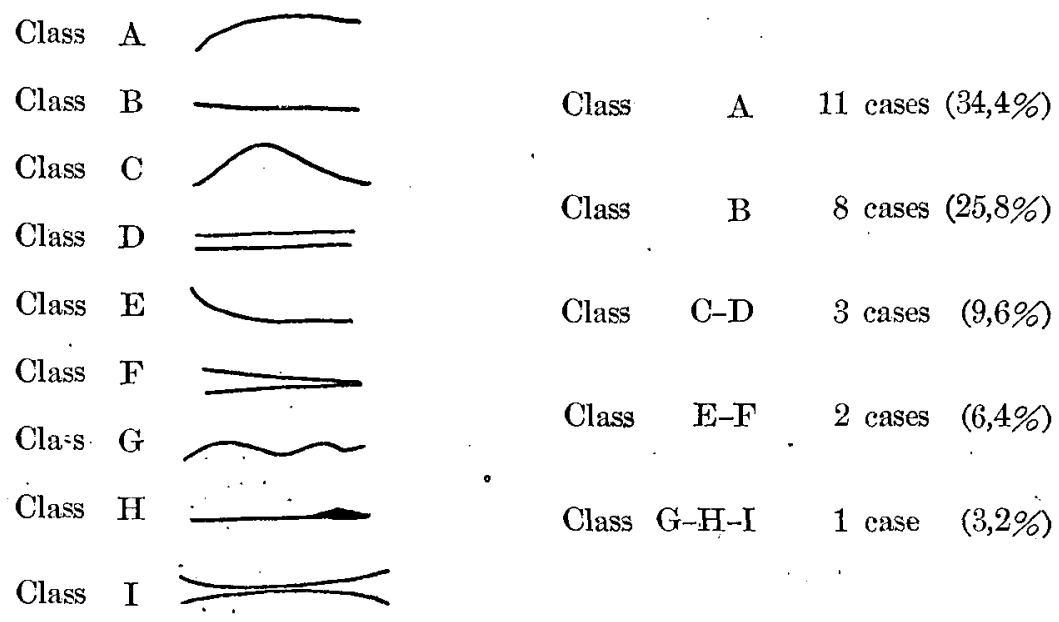

In comparing the location and type of the hairlines and the patholngical changes of the lung space due to disease the following results were found. (Table 2)

\section{Height and Shape of the Diaphragmatical Curve.}

The Levy-Dorn procedure was employed in order to study the height and shape of the diaphragmatical curve. Results are indicated in the following table. (Table 3) 
Table 2.

\begin{tabular}{|c|c|c|c|c|}
\hline \multirow{2}{*}{$\frac{\text { Case }}{1}$} & \multicolumn{2}{|c|}{ Intercostal Spaces } & \multirow{2}{*}{$\frac{\text { Shape }}{\mathrm{E}}$} & \multirow{2}{*}{$\frac{\text { Pathological Changes }}{(-)}$} \\
\hline & VI-VII & III-IV & & \\
\hline 2 & VII-VIII & III-IV & A & $(-)$ \\
\hline 4 & VII-VIII & III-IV & $\mathbf{E}$ & $\begin{array}{l}\text { Upper lobe of the right lung patho- } \\
\text { logical shadow? }\end{array}$ \\
\hline 6 & VII-VIII & III-IV & A & $(-)$ \\
\hline 7 & VIr-VIII & III-IV-V & $\Lambda$ & $(-)$ \\
\hline 11 & VII-VIII & III-IV & I & $(-)$ \\
\hline 12 & VII-VIII & TV-V & A & $(-)$ \\
\hline 14 & VII-VIII & III-IV & G & $(\dot{-})$ \\
\hline 15 & VI-VII & III-IV & B & $\begin{array}{l}\text { Upper lobe of the left lung patho- } \\
\text { logical shadow. }\end{array}$ \\
\hline 16 & VII-VIII & III-IV & $\mathrm{F}$ & Insiltration -left subclavian groove. \\
\hline 19 & V1-VII & III-IV & B & $(-)$ \\
\hline 20 & VI-VII & II-III-IV & $\mathrm{C}$ & Both upper lobes pathological shadow. \\
\hline 21 & VII-VIII & IV $-\mathrm{V}$ & A & $(-)$ \\
\hline 22 & VI-VII & III-IV & $\mathrm{D}$ & $(-)$ \\
\hline 26 & VII-VIII & III-IV & $\mathrm{D}$ & $(-)$ \\
\hline 29 & VIII-IX & IV-V & $\mathbf{B}$ & $(-)$ \\
\hline 30 & VII-VIII & III-IV & $A$ & $(-)$ \\
\hline 32 & VII-VIII & III-IV & $\mathrm{D}$ & $(-)$ \\
\hline 84 & VI-VII & MI-IV & A & Right lower lobe "primüre komplex" \\
\hline 35 & VII-VIII & $I V-V$ & B & $\begin{array}{l}\text { Right apex pathological shadow and } \\
\text { infiltration of the right middle lobe. }\end{array}$ \\
\hline 38 & VI-VII & IV-V & $\mathrm{C}$ & $(-)$ \\
\hline 39 & VII-VIII & III-IV & B & $(-)$ \\
\hline 40 & VI-VII & III-IV & B & Both "Hilus Drïsen" swelling. \\
\hline 41 & VII-VIII & IV $-\mathrm{V}$ & B & $(-)$ \\
\hline 42 & VII-VIII & III-IV & $\mathrm{C}$ & $(-)$ \\
\hline 43 & VI-VII & III-IV & $A$ & $\begin{array}{l}\text { Right apex infiltration. Both "Hilus } \\
\text { Drisien" swelling. }\end{array}$ \\
\hline $4 \overline{5}$ & VII-VIII & III-IV & B & $\begin{array}{l}\text { A plait -inner side of the right dia- } \\
\text { phragma picture. }\end{array}$ \\
\hline 46 & VII-VIII & IV-V-VI & $A$ & Left "Hilus Drïsen" swelling. \\
\hline 47 & VII-VIII & III-IV & A & Right apex tuberclosis. \\
\hline 48 & VI-V1I & III-IV & $\mathrm{F}$ & Left "Hilus Drüsen" swelling. \\
\hline 50 & VI1-VIII & III-IV-V & A & $\begin{array}{l}\text { Pathological shadow -right subclavian } \\
\text { groove? }\end{array}$ \\
\hline 52 & VII-VIII & III-IV & $\mathrm{H}$ & Right under lobe "primiire komplex". \\
\hline
\end{tabular}


Table 3

\begin{tabular}{|c|c|c|c|c|}
\hline Case & $\begin{array}{l}\text { Height } \\
\text { (cme.) }\end{array}$ & $\begin{array}{c}\text { Dorsal } \\
\text { Vertebrae }\end{array}$ & $\begin{array}{l}\text { Distention } \\
\text { of Stomach }\end{array}$ & Shape \\
\hline 1 & 2.4 & $\mathrm{XI}$ & $(-)$ & $\begin{array}{l}\text { Right curve angular, left curve horizontal } \\
\text { then sharp decline to lateral wall. }\end{array}$ \\
\hline 2 & 1.7 & $\mathrm{XI}$ & $(+)$ & Left curve saddle shaped. \\
\hline 3 & 1.1 & $\mathrm{XI}$ & $(-)$ & Right somewhat angular. \\
\hline 4 & 2.2 & XI-XII & $(+)$ & Both sides bow shaped. \\
\hline 5 & 2.5 & $\mathrm{XI}-\mathrm{XII}$ & $(+)$ & Right curve waved. \\
\hline 6 & 1.1 & $\mathrm{XI}$ & $(+)$ & $\begin{array}{l}\text { Right curve bow shaped, left curve saddle } \\
\text { shaped. }\end{array}$ \\
\hline 7 & 1.5 & $\mathrm{XI}-\mathrm{XII}$ & $(+)$ & Both curvesbow shaped. \\
\hline 8 & 1.7 & $\mathrm{XI}-\mathrm{XII}$ & $(+)$ & Both curves bow shaped. \\
\hline 9 & 1.4 & $\mathrm{XI}$ & $(+)$ & $\begin{array}{l}\text { Right curve bow shaped, left curve saddle } \\
\text { shaped. }\end{array}$ \\
\hline 10 & 1.0 & $\mathrm{XI}$ & $(+)$ & $\begin{array}{l}\text { Both sides somewhat horizontal then } \\
\text { sharp decline to lateral wall. }\end{array}$ \\
\hline 11 & 2.4 & $\mathrm{XI}$ & $(+)$ & Both sides bow shaped. \\
\hline 12 & $1 . \dot{5}$ & XI-XII & $(+)$ & Both sides bow shaped. \\
\hline 13 & 2.5 & $\mathrm{XI}$ & $(+)$ & Both sides bow shaped. \\
\hline 14 & - & & & \\
\hline 15 & 1.1 & $\mathrm{X}-\mathrm{XI}$ & $(+)$ & $\begin{array}{l}\text { Right curve bow shaped left curve saddle } \\
\text { shaped. }\end{array}$ \\
\hline 16 & 1.9 & $\mathrm{XI}$ & $(+)$ & Both sides bow shaped. \\
\hline 17 & 1.2 & $\mathrm{XI}-\mathrm{XII}$ & $(-)$ & Both sides bow shaped. \\
\hline 18 & 2.4 & $\mathrm{X}-\mathrm{XI}$ & $o(+)$ & $\begin{array}{l}\text { Right curve deccidedly mount shaped, } \\
\text { left side bow shaped. }\end{array}$ \\
\hline 19 & 3.1 & XI & $(+)$ & $\begin{array}{l}\text { Right curve notched divided into } 2 \text {, left } \\
\text { bow shaped. }\end{array}$ \\
\hline 20 & 2.4 & $\mathrm{XI}-\mathrm{XII}$ & $(+)$ & Both sides straight. \\
\hline 21 & 2.3 & $\mathrm{XI}$ & $(+)$ & $\begin{array}{l}\text { Right side notched twice divided into } 3 \\
\text { parts, left saddle shaped. }\end{array}$ \\
\hline 22 & 1.8 & $\mathrm{x}$ & $(\pi t)$ & Both sides bow shaped. \\
\hline 23 & 1.7 & $\mathrm{XI}-\mathrm{XII}$ & $(+)$ & Both sides bow shaped. \\
\hline 24 & & & & 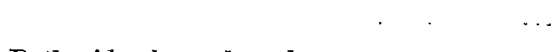 \\
\hline 35 & 1.8 & $\mathrm{XI}$ & $(+)$ & Both sides bow shaped. \\
\hline 26 & 2.5 & $\mathrm{XI}-\mathrm{XII}$ & $(+)$ & Both sides horizontal. \\
\hline 27 & 1.6 & $\mathrm{XI}$ & $(+)$ & Both sides bow shaped. \\
\hline 28 & 2.2 & $\mathrm{X}-\mathrm{XI}$ & $(+)$ & Both sides bow shaped. \\
\hline 29 & 1.8 & $\mathrm{XI}$ & $(+)$ & Both sides bow shaped. \\
\hline 30 & 1.9 & $\mathrm{XI}$ & $(+)$ & Right side notched near the lateral wall: \\
\hline 31 & 0.9 & $\mathrm{XII}$ & $(+)$ & Both sides bow shaped. \\
\hline 32 & 11 & XI-XII & $(+)$ & Both sides bow shaped. \\
\hline 33 & 1.4 & $\mathrm{XI}-\mathrm{XIT}$ & $(+)$ & Right curve bow shaped, left saddle shaped. \\
\hline 34 & 1.6 & $\mathrm{X}-\mathrm{XI}$ & $(+)$ & $\begin{array}{l}\text { Left side the outer } 1 / 3 \text { makes a "höckerig" } \\
\text { upward. }\end{array}$ \\
\hline $3 \tilde{a}$ & 2.2 & $\mathrm{X}-\mathrm{XI}$ & $(+)$ & $\begin{array}{l}\text { Right curve notched divided into } 2 \text {, left } \\
\text { saddle shaped. }\end{array}$ \\
\hline
\end{tabular}




\begin{tabular}{|c|c|c|c|c|}
\hline Case & $\begin{array}{l}\text { Height } \\
\text { (cm.) }\end{array}$ & $\underset{\text { Vorsal }}{\text { Vertebrae }}$ & $\begin{array}{l}\text { Distention } \\
\text { of Stomach }\end{array}$ & $\therefore \quad$. \\
\hline 36 & . & & $\because$ & \\
\hline 37 & 1.2 & $\mathrm{XI}$ & $(-)$ & Both sides bow shaped. \\
\hline 38 & 1.8 & $\mathrm{XI}$ & $(+)$ & Both sides bow shaped. \\
\hline 39 & 2.1 & $\mathbf{X I}-\mathbf{X I I}$ & $(-)$ & $\begin{array}{l}\text { Right side somewhat straight, left hori- } \\
\text { zontal then sharp decline to lateral wall. }\end{array}$ \\
\hline 40 & Anclear & & & \\
\hline 41 & 0.7 & $\mathrm{X}-\mathrm{XI}$ & $(+)$ & $\begin{array}{l}\text { Right curve deccidedly mount shaped in } \\
\text { center. }\end{array}$ \\
\hline & $\cdot$ & & & Left bow shaped. \\
\hline 42 & 1.6 & $\mathrm{XI}$ & $(+)$ & $\begin{array}{l}\text { Left side straight, then sharp decline to } \\
\text { lateral wall. }\end{array}$ \\
\hline 43 & 1.2 & $\mathbf{X I}$ & $(-)$ & Both sides bow shaped. \\
\hline 44 & 2.3 & $\mathrm{X}-\mathrm{XI}$ & $(+)$ & Both sides bow shaped. \\
\hline 45 & 2.6 & $\mathrm{X}-\mathrm{XI}$ & $(+)$ & $\begin{array}{l}\text { Right side the inner } 1 / 3 \text { makes a } \\
\text { " höckerig" upward. }\end{array}$ \\
\hline 46 . & 2.6 & $\mathrm{X}-\mathrm{XI}$ & $(+)$ & $\begin{array}{l}\text { Spine shaped deviation a. center of right } \\
\text { curve, left saddle shapedt }\end{array}$ \\
\hline 47 & 2.5 & $\mathrm{XI}$ & $(-)$ & $\begin{array}{l}\text { Right curve notched divided into } 3 \text {, left } \\
\text { saddle shaped. }\end{array}$ \\
\hline 48 & Anclear & & & \\
\hline 49 & 2.1 & $\mathrm{XI}$ & $(-)$ & Both sides bow shaped. \\
\hline 50 & 2.1 & $\mathrm{XI}$ & $(-)$ & Both sides bow shaped. \\
\hline 51 & 1.7 & $\mathrm{x}$ & $(+)$ & $\begin{array}{l}\text { Right curve notched divided into } 2 \text {, left } \\
\text { bow shaped. }\end{array}$ \\
\hline 52 & 2.5 & $\mathrm{XI}$ & $(-) 0$ & Right curve notched divided into 3 . \\
\hline 53 & $2.0^{\circ}$ & $\mathrm{XI}-\mathrm{XII}$ & $(-)$ & Both sides bow shaped. \\
\hline 54 & 1.4 & $\mathrm{X}-\mathrm{XI}$ & $(+)$ & $\begin{array}{l}\text { Right curve mount shaped near heart, } \\
\text { left bow shaped. }\end{array}$ \\
\hline
\end{tabular}

\section{X-ray of Heart and}

\section{Blood Vessels.}

The measurements were made according to Prof. Dr. Saito and Dr. Aikawa's . method. See following diagram and table:

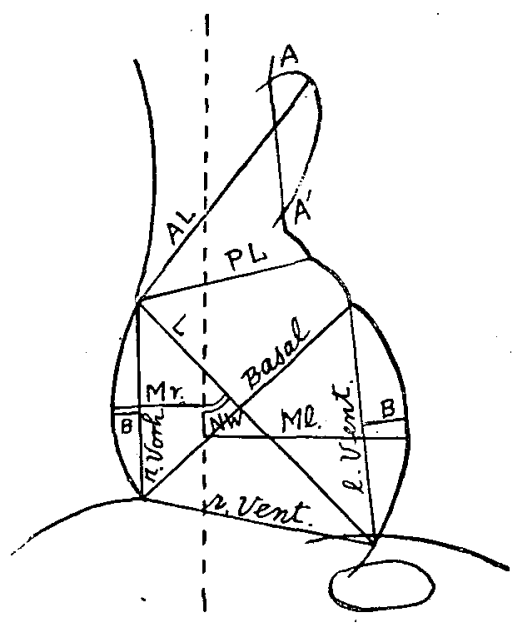


Table 4.

\begin{tabular}{|c|c|c|c|c|c|c|c|c|c|c|c|c|}
\hline No. & N.W. & L. & Mr. & MI. & Mr. +1 & r. Vorh. & r. Vent. & 1. Vent. & Basal & Pl. & Al. & $A-A^{\prime}$ \\
\hline 1 & 38 & 16.3 & 4.2 & 9.1 & 13.3 & $9.2(1.4)$ & 11.0 & $10.1(1.9)$ & 11.8 & 7.6 & 8.8 & 3.2 \\
\hline 2 & 41 & 15.2 & 4.0 & 8.3 & 12.3 & $9.2(1.7)$ & 10.2 & $11.4(1.7)$ & 1.1 .2 & .5 .9 & 8.4 & $: 3.2$ \\
\hline 3 & 43 & 14.4 & 4.6 & 8.0 & 12.6. & $8.2(1.0)$ & 10.8 & $10.4(2.4)$ & 11.4 & 7.0 & 9.6 & 3.8 \\
\hline 4 & 40 & 16.1 & 4.3 & 9.2 & 13.5 & $9.8(1.8)$ & 11.0 & $9.9(1.6)$ & 12.1 & .6 .6 & 8.1 & 3.7 \\
\hline 5 & 46 & 16.2 & 5.1 & 10.2 & 15.3 & $9.1(2.0)$ & 12.0 & 10.0 & 13.2 & 7.1 & 10.3 & 3.0 \\
\hline 6 & 40 & 15.0 & 5.3 & 7.4 & 12.7 & $9.4(1.4)$ & 10.4 & $9.3(1.7)$ & 12.1 & 7.8 & 10.0 & 3.1 \\
\hline 7 & 35 & 13.2 & 4.6 & 5.8 & 10.4 & $9.6(1.1)$ & 7.7 & $10.3(2.1)$ & 11.3 & 6.5 & 9.3 & 3.2 \\
\hline 8 & 43 & 14.3 & 4.0 & 7.8 & 11.8 & $9.1(1.4)$ & 9.0 & $11.2(2.0)$ & 11.6 & 7.4 & 11.5 & 2.4 \\
\hline 9 & 45 & 15.3 & 4.1 & 9.3 & 13.4 & $8.3(1.2)$ & 12.0 & $10.3(1.9)$ & 12.0 & 7.0 & 8.8 & 2.5 \\
\hline 10 & 48 & 15.0 & 4.2 & 9.3 & 13.5: & $8.6(1.6)$ & 12.1 & $9.1(1.3)$ & 11.6 & 6.4 & 8.8 & 4.6 \\
\hline 11 & 50 & $15.1^{\circ}$ & 4.7 & 8.5 & 13.2 & $6.7(0.9)$ & 12.0 & $10.4(2.0)$ & 11.2 & 7.7 & 9.8 & 4.3 \\
\hline 12 & 42 & 14.0 & 4.3 & 7.8 & 12.1 & $8.8(1.6)$ & 8.5 & $10.3(1.8)$ & 11.8 & 7.6 & 9.7 & 3.2 \\
\hline 13 & 39 & 14.6 & 4.5 & 7.0 & 11.5 & $8.1(1.1)$ & 10.5 & $12.0(1.9)$ & 10.2 & 6.5 & 11.0 & ? \\
\hline 14 & 45 & 15.7 & 5.1 & 7.9 & 13.0 & $8.7(1.3)$ & 11.7 & $9.9(1.7)$ & 12.0 & 7.0 & 8.8 & 4.8 \\
\hline 15 & 42 & 14.8 & 4.2 & 8.1 & 12.3 & $9.3(2.0)$ & 9.6 & $11.0(1.6)$ & 11.5 & 6.5 & 8.2 & 3.2 \\
\hline 16 & 47 & 14.2 & 4.3 & 8.2 & 12.5 & $6.5(0.8)$ & 10.7 & $10.8(2.2)$ & 10.8 & 7.2 & 9.3 & 3.9 \\
\hline 17 & 40 & 15.1 & 3.2 & 9.2 & 12.4 & $9.9(1.5)$ & 10.1 & $10.0(1.8)$ & 11.4 & 5.3 & 7.9 & 2.4 \\
\hline 18 & 48 & 16.4 & 4.1 & 10.4 & 14.5 & $8.5(0.2)$ & 13.4 & $9.5(2.7)$ & 11.8 & 7.5 & 8.8 & 2.3 \\
\hline 19 & 39 & 15.1 & 3.8 & 8.3 & 12.1 & $8.7(1.4)$ & 10.3 & $11.6(2.0)$ & 11.3 & 6.6 & 9.9 & 2.2 \\
\hline 20 & 53 & 13.2 & 3.8 & 8.1 & 11.9 & $8.4(1.5)$ & 9.9 & $10.2(1.5)$ & 10.3 & 6.5 & 9.3 & 4.1 \\
\hline 21 & 48 & 14.1 & 4.4 & 8.7 & 13.1 & $8.9(1.8)$ & 9.0 & $10.6(2.1)$ & 12.0 & 7.8 & 9.9 & 4.2 \\
\hline 22 & 46 & 15.7 & 4.5 & 9.0 & 13.5 & $7.9(1.1)$ & 13.2 & $10.0(1.5)$ & 10.2 & 5.8 & 7.4 & 2.8 \\
\hline 23 & 47 & 18.8 & 3.9 & 9.1 & 13.0 & $7.6(1.2)$ & 11.5 & $11.6(1.7)$ & 11.4 & 7.5 & 9.7 & 3.9 \\
\hline 24 & 40 & 14.1 & 4.9 & 8.0 & 12.9 & $7.6(1.7)$ & 9.2 & $11.2(2.0)$ & 10.1 & 6.6 & 8.5 & 3.6 \\
\hline 25 & 44 & 15.5 & 4.1 & 9.4 & 13.5 & $9.1(1.7)$ & 11.4 & $11.8(1.9)$ & 11.9 & 8.5 & 9.0 & 4.0 \\
\hline 26 & 44 & 15.4 & 4.7 & 9.2 & 13.9 & $8.5(1.7)$ & 11.2 & $9.4(1.9)$ & 11.7 & 7.2 & 9.5 & 4.3 \\
\hline 27 & 43 & 15.8 & 3.7 & 9.4 & 13.1 & $8.0(1.3)$ & 11.8 & $12.3(2.0)$ & 11.8 & 6.9 & 9.5 & 3.9 \\
\hline 28 & 39 & 12.7 & 3.3 & 7.2 & 10.5 & $8.0(0.9)$ & 9.5 & $11.6(1.9)$ & 11.0 & 5.6 & 8.5 & 3.5 \\
\hline 29 & 43 & 16.0 & 4.5 & 9.0 & 13.5 & $8.9(1.7)$ & -10.9 & $10.3(1.6)$ & 11.5 & 6.9 & 9.6 & .2 .5 \\
\hline 30 & 45 & 13.2 & 4.0 & 6.5 & 10.5 & $7.0(0.6)$ & 10.4 & $12.5(1.6)$ & 11.8 & 6.8 & 10.0 & 3.0 \\
\hline 31 & 38 & 14.8 & 5.2 & 7.4 & 12.6 & $9.9(2.0)$ & 9.4 & $13.0(2.3)$ & $12.1 \%$ & 5.8 & 8.5 & 3.0 \\
\hline 32 & 44 & 13.8 & 4.9 & 8.0 & 12.9 & $7.7(1.0)$ & 11.2 & $12.1(2.3)$ & 12.9 & 7.1 & 8.0 & 2.5 \\
\hline 33 & 36 & 15.4 & 4.0 & 8.0 & 12.0 & $9.8(1.1)$ & 10.3 & $11.0(1.9)$ & 12.7 & 5.6 & 7.9 & 3.1 \\
\hline 34 & 45 & 14.8. & 5.4 & 7.4 & 12.0 & $8.3(1.2)$ & 10.7 & $12.5(2.3)$ & 12.2 & 7.3 & 9.4 & 3.4 \\
\hline $\begin{array}{l}35 \\
36\end{array}$ & .54 & 14.5 & 5.5 & 8.0 & 13.5 & $5.5(0.6)$ & 12.4 & $11.7(2.1)$ & 12.0 & 8.6 & 10.4 & 3.5 \\
\hline 37 & 49 & 14.9 & 4.6 & 8.9 & 13.5 & S.I (1.5) & 11.4 & $11.7(1.7)$ & 12.0 & 7.1 & 9.9 & 3.3 \\
\hline 38 & 44 & 14.5 & 4.4 & 9.8 & 14.2 & $7.8(1.3)$ & 11.2 & $10.0(2.7)$ & 12.2 & 7.4 & 9.9 & 3.5 \\
\hline 39 & 41 & 14.9 & 4.3 & 8.8 & 13.1 & $7.8(1.0)$ & 10.8 & $13.6(3.2)$ & 12.6 & 7.7 & 10.9 & 3.3 \\
\hline 40 & 56 & 12.5 & 5.2 & 6.7 & 11.9 & $9.7(1.6)$ & 9.4 & $10.5(1.4)$ & 11.5 & 6.6 & 9.1 & 3.0 \\
\hline 41 & 44 & 15.9 & 6.5 & 8.1 & 14.6 & $10.1(2.2)$ & 12.0 & $13.9(2.3)$ & 14.1 & 7.8 & 10.0 & 2.5 \\
\hline 42 & 44 & .14 .7 & 4.3 & 6.5 & 10.8 & $9.1(1.2)$ & 9.7 & $10.4(1.8)$ & $\cdot 12.4$ & 7.1 & 10.8 & 4.0 \\
\hline
\end{tabular}




\begin{tabular}{|c|c|c|c|c|c|c|c|c|c|c|c|c|}
\hline No. & M.W. & I. & Mr. & Ml. & $\mathrm{Mr} .+1$ & r. Vorh. & r. Vent. & 1. Vent. & Basal & $\mathrm{Pl}$ & Al. & $A-A^{\prime}$ \\
\hline 43 & 38 & 13.5. & 4.0 & 7.4 & 11.4 & $9.6(1.5)$ & 8.8 & $10.2(2.0)$ & 11.3 & 6.2 & 8.8 & 2.7 \\
\hline 44 & 44 & 14.6 & 3.1 & 8.0 & 11.1 & $8.2(0.9)$ & 9.9 & $11.6(1.3)$ & 11.2 & 7.7 & 12.0 & 2.8 \\
\hline 45 & 44 & 14.4 & 3.5 & 8.9 & 12.4 & $6.6(0.6)$ & 11.4 & $11.0(1.7)$ & 11.3 & 7.2 & 9.4 & 3.0 \\
\hline 46 & 42 & 14.1 & 4.0 & 9.0 & 13.0 & $8.4(1.2)$ & 10.5 & $11.1(2.6)$ & 11.5 & 6.2 & 8.5 & 4.2 \\
\hline 47 & 37 & 14.9 & 5.4 & 7.4 & 12.8 & $8.8(1.5)$ & 10.9 & $11.3(2.2)$ & $11 . \mathrm{S}$ & 6.3 & 8.1 & 4.1 \\
\hline 48 & 43 & 15.5 & 4.5 & 8.2 & 12.7 & $7.3(0.7)$ & 11.3 & $11.5(2.0)$ & 11.6 & 8.2 & 10.9 & $+?$ \\
\hline 49 & 43 & 13.9 & 3.3 & 8.1 & 11.4 & $7.8(1.0)$ & 10.8 & $9.5(1.0)$ & 10.8 & 6.1 & 8.8 & 4.0 \\
\hline 50 & 48 & 14.4 & 3.5 & 8.5 & 12.0 & $7.8(1.0)$ & 10.2 & $9.7(1.3)$ & 11.2 & 7.4 & 10.0 & 3.3 \\
\hline 51 & .46 & 15.4 & 4.6 & 10.2 & 14.8 & $8.5(2.0)$ & 12.4 & $9.8(2.3)$ & 11.3 & 5.5 & 8.2 & $?$ \\
\hline 5 & 40 & 15.0 & 4.8 & 8.9 & 13.7 & $8.0(1.4)$ & 10.6 & $10.2(2.1)$ & 10.6 & 7.3 & 10.6 & 3.7 \\
\hline 53 & 48 & $13 . \tilde{5}$ & 3.8 & 7.5 & 11.3 & $8.0(0.9)$ & 9.7 & $9.4(1.1)$ & 11.2 & 7.5 & 12.0 & 3.9 \\
\hline 54 & 52 & 13.1 & 4.8 & 8.8 & 13.6 & $\cdot 7.7(1.6)$ & 11.8 & $8.6(1.4)$ & 10.7 & 5.1 & 6.9 & 3.0 \\
\hline
\end{tabular}

\section{Conclusion.}

Part.. 1. Clavicle.

A. "Begleitschatten" of the Clavicle.

It was determined and elearly secn in $86.8 \%$ of the cascs and in greater part on both sides.

B. "Usurschatten" of the Clavicle.

The condition occurred in $24.5 \%$ of the subjects: If the "doubtful" case: were included, the percentage, would be increased to $45.3 \%$. In considering the movements involed in playing tenuis, this state was one to be expected.

C. Epiphy:es.

This state exi ted in 56.6\% of the examined. In comparing the age range with the occurrence, the relationship concured with previous reports.

Part 2. Ribs.

A. Ossification.

Complete ossification of one or more ribs occurred in $32.1 \%$ of the players.

1) Ossification of the first rib cartilage was in general very marked.

2) The ossification of the first rib in comparison with that of the second and lower rib was more complete and marked.

3) In general the state was in direct proportion to the age but exceptions to the rule might occur.

4) In considering the relationship between the pathological changes present in the ling field and the state of ossification, it is interesting to note that 9 of the 17 cases (53\%) 
showed no changes. 6 cases (35\%) had indications of changés and $2(12 \%)$ were doubtful. I believe that this present a problem for further research.

B. Rib Deformities of the deviations noted 4 cases may be considered rib deformities. They occurred mainly on the right side. 2 cases may be considered deformities involving the "aligument" of the ribs.

Part 3. "Begleitschatten" of the second rib.

- It was found to exist in about half of the subjects examined:

1) Among which the greater proportion may be considered normal.

2) In the few abnormal cases there was an accompanying pathological change;

3) The presence of a diseased condition did not necessarily have an accompaning "Begleitschatten";

4) Even though the "Begleitschatten" may be considered normal, it did not follow there was no pathological condition existant.

Part 4. Hair-line. (Right inter-lobes pleura picture:)

Although found in $60.4 \%$ of the players, with the exception of the very musual cases there seemed to be no relationship to pathological conditions of the Iungs.

Part. 5. Height aud Shape of the Diaphragmatical Curve.

In the greater number of cases the right curve was higher than the left. It was interesting to unte that notches appeared in 7 cases but only on the right side.

Part 6. Heart and Blood Vessels.

1) The enlargement of the heart of the temis players was on a par with that of the " model" sportsman; in fact greater in several cases.

2) There was no comparison possible of the enlargement of the heart between that of a non-participant and that of a temis player an indication of the rigorous training required of an excellent temis player.

3) The Vena Cava Inferior appeared less frequently on the $\mathrm{X}$-ray photograph than that of the "model" sportsman.

4) The appearance of the Vena Cava Superior and Inferior and the arch of the pulmonary vein on the photograph indicated that the "Lungen Zeichumg" was clear.

- Part 7. Pathological Changes of the Lumgs.

$22.6 \%$ of the subjects had clear indications of such conditions. If the "borderline" cases were to be included, the percentage mounted to 39.6\%. Although this problem must await further research, I an led to believe that those of relatively weaker constitutions are partici- 
pating in temuis. They may have chosen tennis, because at a glance it may appear to be a less rigorous game than other sports. The fact that in the greater number of cases the $x$-ray findings indicate that the pathological changes are of long-standing further substantiates the foregoing statement.

With the exception of the lungs there was no outstanding difference in the X-ray findings of those of temuis players and the participants of other sports.

\section{日 本 醫 學 雜 誌 婄關係雑誌目錄支略名表}

雜 婄 名

Niigata Ika Daigaku Byôrigaku Kyoshitsu

Kenkyû Hôkoku. （新潟醫科大學病理學教 定研究夁告)

Nisshin Chiryô.（日新治療）

Nisshin Igaku.（日新醫學）

Nò. (腦)

Nôhi Igakkai Zasshi.（浱飛醫學會雜誌）

Nyûjigaku Zasshi.（乳兒學雜誌）

Nyâyôji Kenkyô. (乳幼兒㸴茫)

\section{$\mathbf{O}$}

Ogata-Byôin Iji Kenkyûkai Iji Kaihô.（緒方 病院覧事研究會醫事會報)

Okayama Igakkai Zasshi. (剬山醫學會雜誌)

Osaka Igakkai Zasshi. (大阪篦學會雜誌)

Osaka Igaku Kenkyûkai 7asshi.（大阪醫學研 究會雜誌)

Osaka Iji Kôron. (大阪醫事公諭)

Osaka Iji Shinshi. (大阪䣽事新誌)

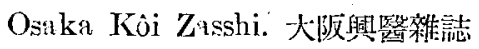

Oto-Rhino-Laryngologia. (耳與咽喉科)

(The) Oto-Rhino-and Laryngological Clinic.

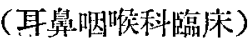

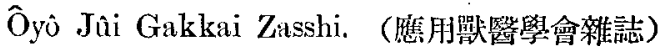

略 名

Niigata Byôri. Hk.

（新潟病理報）

Nisshin Chiryô (日新治療)

Nisshin 1g. (日新醫學)

Nô (腦)

Nôhi Igk. Z. (瀑飛醫會誌)

Nyâji. Z. (乳兒誌)

Nyûyôji Kenkyû (乳约兒砩究)

Ogata. Iji. Kh. (緒方醫事會報)

Okayama Tgk. Z. (風山醫會誌)

Osaka Igk. Z. (阪醫會誌)

Osaka Ig. Kenkyû. Z. (阪醫砳誌)

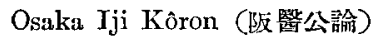

Osaka Iji Ss. (阪醫筷誌)

Osaka Kôi Z. (阪興矮誌)

Otologia. (Fukuoka)

(Otol. Cl.) $($ Kyoto $)=$ Jibi. Rinshô

Ôyô Jûi Gk. Z. (應朋獸醫會誌) 